\title{
On-chip green silica upconversion microlaser
}

\author{
Tao Lu, ${ }^{1}$ Lan Yang, ${ }^{1,3}$ Rob V. A. van Loon, ${ }^{2}$ Albert Polman, ${ }^{2}$ and Kerry J. Vahala ${ }^{1, *}$ \\ ${ }^{1}$ Applied Physics Department, California Institute of Technology, 1200 E. California Boulevard, \\ Pasadena, California 91125, USA \\ ${ }^{2}$ Center for Nanophotonics, Foundation for Fundamental Research on Matter Institute for Atomic and Molecular \\ Physics, Kruislaan 407, 1098 SJ Amsterdam, The Netherlands \\ ${ }^{3}$ Present address: Department of Electrical and System Engineering, Washington University in St. Louis, \\ St. Louis, Missouri 63130, USA \\ *Corresponding author: vahala@caltech.edu
}

Received November 5, 2008; revised January 7, 2009; accepted January 8, 2009; posted January 15, 2009 (Doc. ID 103577); published February 11, 2009

\begin{abstract}
We demonstrate an erbium-doped silica toroidal microcavity upconversion laser on a silicon chip lasing in the visible spectral range $(510-580 \mathrm{~nm})$. The microcavity is pumped at $1458 \mathrm{~nm}$ by a tapered optical fiber coupled to the cavity and the lasing threshold is $690 \mu \mathrm{W}$. Lasing is observed at room temperature despite the high nonradiative relaxation rates of Er in pure silica that usually precludes upconversion lasing from higher excited states. This is attributed to the very high circulating pump power in the high- $Q$ microcavity $\left(Q>10^{7}\right)$. ( 2009 Optical Society of America

OCIS codes: $140.3613,140.3500,140.7300$.
\end{abstract}

The design and fabrication of a silicon-based laser operating in the visible, and integrated with optical and electronic components on a single chip, is one of the main challenges in silicon-based optoelectronic technology [1]. Such a laser sees critical applications in areas, such as on-chip biodetection, and must be integrated with fiber and/or planar waveguide architectures on Si. Previously, on-chip IR Si-based Er-doped microcavity lasers have been demonstrated [2-4], lasing near $1.5 \mu \mathrm{m}$ when pumped using a $1460 \mathrm{~nm}$ pump laser.

One way to achieve green laser action from an Erdoped material is by using upconversion. Upconversion lasers operate through the excitation of a higherlying state either by absorption of multiple lowerenergy pump photons [5] or by cooperative upconversion due to dipole-dipole interaction between excited ions [6]. Rare-earth-doped glasses are ideal hosts for upconversion lasers due to the relatively long lifetimes of the $4 f$ manifolds. A large effort has been put into engineering Er-doped glasses with minimal phonon energies and hence long excitedstate lifetimes. Indeed, green upconversion lasing has been observed in $\mathrm{ZrF}_{4}-\mathrm{BaF}_{2}-\mathrm{LaF}_{3}-\mathrm{AlF}_{3}-\mathrm{NaF}$ (ZBLAN) compound glasses [7-9], where Klitzing et al. [10] have reported a green upconversion laser with a threshold power as low as $30 \mu \mathrm{W}$ using a ZBLAN microsphere as the resonant cavity.

The ZBLAN material, however, is incompatible with $\mathrm{Si}$ processing technology. Pure silica, on the other hand, has proven to be an excellent material to fabricate on-chip microcavities [11,12], but so far, the short excited-state manifold lifetime that results from the relatively high phonon energy in silica has precluded the observation of upconversion lasing in such cavities. In this Letter, we demonstrate a way to circumvent this limitation of the excited-state transition level in a silica host by using a resonant cavity with an extremely high quality factor $\left(Q>10^{7}\right)$. The resonator device is fabricated on a silicon wafer enabling integration of the green lasing emission with optoelectronic functionality on the same chip.
Er-doped silica microcavities were fabricated on $\mathrm{Si}$ substrates using a sol-gel process [11,12]. The typical toroid major and minor diameters measured 40 and $4 \mu \mathrm{m}$, respectively. The inset of Fig. 1(a) shows a scanning electron microscope image of an Er-doped toroidal microcavity. The typical Er concentration in the cavity is estimated to be $0.1-0.5$ at. \%. The typical intrinsic quality factor of these Er-doped cavities [4] is well above $10^{7}$. For a toroidal cavity with an intrinsic quality factor of $Q=5 \times 10^{7}$ a pump power of $10 \mathrm{~mW}$ coupled into the cavity will build up a circulating power as high as $1 \mathrm{~kW}$. This circulating power is concentrated into a cross sectional area of only several square micrometers, giving rise to an intensity that can exceed $1 \mathrm{GW} / \mathrm{cm}^{2}$. As will be demonstrated below, such high intracavity intensities are sufficient to create population inversion of higher-lying excited states of $\mathrm{Er}^{3+}$, which is otherwise difficult to achieve due to their fast relaxation rates.

To characterize the optical quality of the Er-doped material, photoluminescence decay measurements were taken using a scanning confocal microscope. Excitation was provided with a $514 \mathrm{~nm} \mathrm{Ar}^{+}$laser. Figure 1(a) shows decay traces taken for the ${ }^{4} I_{13 / 2}$ $\rightarrow{ }^{4} I_{15 / 2}$ transition at $1550 \mathrm{~nm}$ and the ${ }^{4} I_{3 / 2} \rightarrow{ }^{4} I_{15 / 2}$ transition at $550 \mathrm{~nm}$. The luminescence decay time at $1550 \mathrm{~nm}$ is $\tau=13 \mathrm{~ms}$, close to the radiative decay time in pure silica glass of $\tau=18 \mathrm{~ms}$ [13]. This indicates a high internal quantum efficiency of the Er ions in the silica host. The deviation from the ideal radiative decay time may be due to concentration quenching of hydroxide (OH) impurities [14]. The lifetime of the $550 \mathrm{~nm}$ level was shorter than the measurement resolution of $\tau=10 \mu \mathrm{s}$. This is consistent with data found earlier for silica glasses that reported a lifetime of $\tau=0.7 \mu \mathrm{s}$ [15]. It is also significantly shorter than the corresponding lifetime in lowphonon energy hosts typically used for upconversion lasing, such as ZBLAN ( $\tau=0.55 \mathrm{~ms})$ [10].

To perform lasing measurements, pump laser light $(\lambda=1450 \mathrm{~nm})$ is coupled into a microcavity using a 


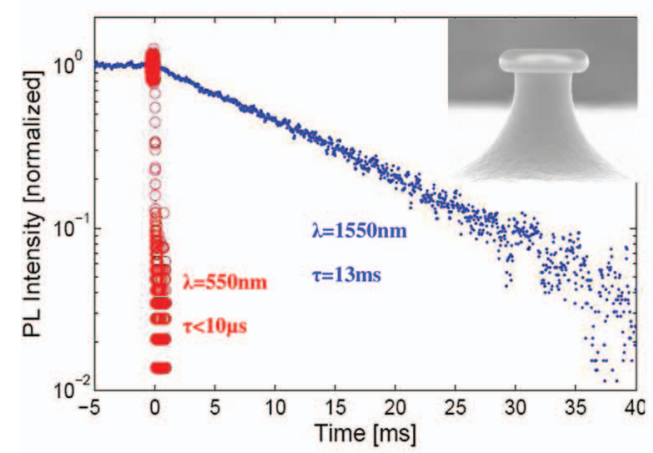

(a)

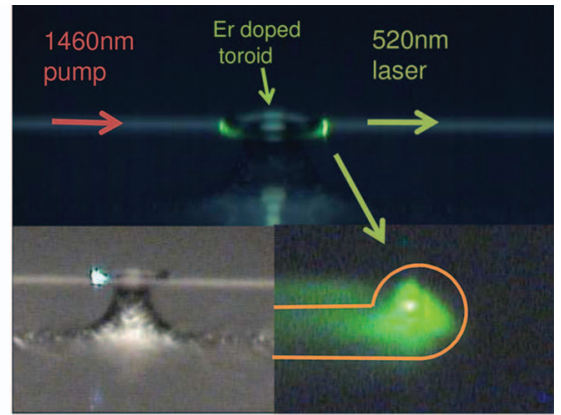

(b)

Fig. 1. (a) Photoluminescence decay measurements of the Er-doped cavity after excitation with a $514 \mathrm{~nm}$ pump pulse, taken for emission wavelengths of 1550 and $550 \mathrm{~nm}$. The decay time from the upconversion level at $550 \mathrm{~nm}$ is less than $10 \mu \mathrm{s}$. The inset is a scanning electron microscope image of the Er-doped silica toroidal microcavity on Si. (b) Optical image of pumped Er-doped cavity showing bidirectional upconversion lasing. The pump fiber is visible as the horizontal green line behind the cavity. The inset at the lower left corner is an optical image of a pumped pure silica cavity showing unidirectional third-harmonic emission.

tapered optical taper that is positioned near the cavity using a piezoelectrically driven stage. The coupling theory is presented in [16], and the taper technique is described in $[17,18]$. The laser emission is coupled out through the same fiber and analyzed using a spectrometer. Furthermore, by adjusting the taper-toroid coupling gap it was possible to fully suppress lasing in the $1500 \mathrm{~nm}$ band as longer wavelength light experiences a larger coupling loss than a shorter wavelength at a given coupling gap. The inset in Fig. 2(a) shows a typical laser emission spectrum collected in the $510-580 \mathrm{~nm}$ spectral range. The emission is highly multimode, and two main emission peaks near 525 and $550 \mathrm{~nm}$ are observed, corresponding to the ${ }^{2} H_{11 / 2} \rightarrow{ }^{4} I_{15 / 2}$ and ${ }^{4} S_{3 / 2} \rightarrow{ }^{4} I_{15 / 2}$ transitions in $\mathrm{Er}^{3+}$. The free spectral range in the spectrum is around $1 \mathrm{~nm}$, in good correspondence with the toroidal cavity's major diameter.

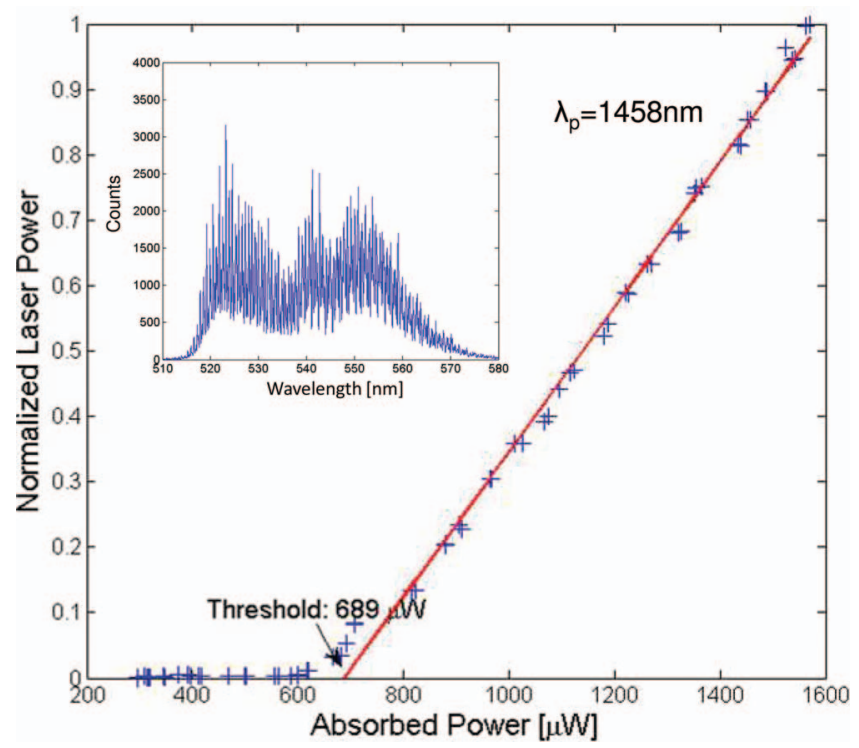

Fig. 2. Normalized upconversion lasing power (integrated in the range $\lambda=510-580 \mathrm{~nm}$ ) as a function of absorbed pump power $\left(\lambda_{p}=1458 \mathrm{~nm}\right)$. The lasing threshold is $690 \mu \mathrm{W}$. The inset shows the multimode lasing spectrum.
The main panel in Fig. 2 shows the laser output power, normalized to the maximum power, integrated over the 510-580 $\mathrm{nm}$ spectral range plotted versus the absorbed pump power at $1458 \mathrm{~nm}$. A clear lasing threshold is observed around $690 \mu \mathrm{W}$, and a linear behavior is observed above the threshold. An optical micrograph of the laser cavity under green upconversion lasing conditions is shown in Fig. 1(b), taken using a CCD camera mounted onto an optical microscope. The green emission is clearly visible in the output fiber and along the full perimeter of the cavity, corresponding to both clockwise and counterclockwise lasing modes. In [19], unidirectional green third-harmonic emission at $517.4 \mathrm{~nm}$ was reported under $1553.9 \mathrm{~nm}$ pumping. For comparison, the inset at the lower left corner of Fig. 1(b) shows an optical micrograph of a pure silica cavity (i.e., without $\mathrm{Er}$ ) emitting green third-harmonic light. The distinct difference in propagation characteristics (bidirectional as opposed to unidirectional) and also the existence of a lasing threshold in the present case indicates that the green emission observed from the Er-doped silica cavity is due to lasing from levels populated by upconversion and not through the third-harmonic process.

The large difference in the wavelength between the pump and the laser emission bands has a strong effect on the spatial overlap of pump and emission modes. The pump mode determines the spatial profile of inverted Er ions inside the cavity. The effective laser gain (including this spatial overlap factor) is then determined by the overlap between the excited Er distribution and the lasing mode. To investigate this, an overlap factor defined as $\Gamma$ $=\int_{\text {core }} \boldsymbol{I}_{p} \boldsymbol{I}_{s} \mathrm{~d} s / \sqrt{\int_{\text {core }} \boldsymbol{I}_{p}^{2} \mathrm{~d} s \int_{\text {core }} \boldsymbol{I}_{s}^{2} \mathrm{~d} s} \quad$ was calculated $[20,21]$. Figures $3(\mathrm{a})$ and $3(\mathrm{~b})$ show the fundamental transverse mode of the pump at $1457 \mathrm{~nm}$ and the fundamental lasing mode at $520 \mathrm{~nm}$, respectively. Clearly, the latter is centered more outward in the cavity relative to the $1457 \mathrm{~nm}$ mode. The calculated overlap factor for these two modes is 0.73 . Mode profile calculations were also performed for several 

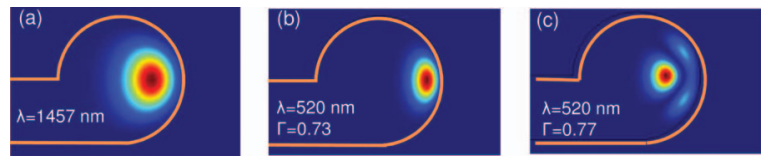

Fig. 3. Calculated mode profiles for a toroidal silica microcavity with a minor radius of $2 \mu \mathrm{m}$ and a major radius of $20 \mu \mathrm{m}$. (a) Fundamental pump mode at $1457 \mathrm{~nm}$. (b) Fundamental lasing mode at $520 \mathrm{~nm}$ showing overlap factor $(\Gamma=0.73)$. (c) Higher-order lasing mode at $520 \mathrm{~nm}$ showing improved overlap $(\Gamma=0.77)$ with the pump mode at $1457 \mathrm{~nm}$ in (a).

higher-order modes at $520 \mathrm{~nm}$, and for each mode the mode overlap factor was calculated with the fundamental pump mode. A larger overlap factor $(0.77)$ was found for the first-order transverse $(q, l)=(1,0)$ laser mode plotted in Fig. 3(c). Here $q, l$ are radial and polar mode numbers following the symbol convention in [21]. The larger overlap factor is consistent with the observation of more complex spatial profiles in high magnification images of emission from the whispering gallery.

In conclusion, we have demonstrated a silica microcavity upconversion laser on a $\mathrm{Si}$ substrate. Multimode laser action is observed in the $510-580 \mathrm{~nm}$ spectral range with a threshold as low as $690 \mu \mathrm{W}$ when pumped at $1458 \mathrm{~nm}$. This Letter demonstrates that, despite the short intrinsic lifetime of the higher excited states of $\mathrm{Er}^{3+}$ in a high-phonon energy host, such as silica, lasing action can be achieved in a high- $Q$ cavity. The next challenge is to integrate the green microlaser with other functionality on the same Si chip [22].

The Dutch part of this work is part of the research program of the Foundation for Fundamental Research on Matter (FOM), the SmartMix program of the Dutch Ministry of Economic Affairs, and the Air Force of Scientific Research (AFOSR), Multidisciplinary University Research program (MURI) award FA9550-05-1-0450. The Caltech part of this work is supported in part by the Defense Advanced Research Projects Agency (DARPA) and the Caltech Lee Center. Tao Lu is partially financially supported by a
Natural Sciences and Engineering Research Council of Canada (NSERC) postdoctoral fellowship.

\section{References}

1. B. Jalali and S. Fathpour, J. Lightwave Technol. 24, 4600 (2006).

2. L. Yang, D. K. Armani, and K. J. Vahala, Appl. Phys. Lett. 83, 825 (2003).

3. A. Polman, B. Min, J. Kalkman, T. J. Kippenberg, and K. J. Vahala, Appl. Phys. Lett. 84, 1037 (2004).

4. L. Yang, T. Carmon, B. Min, S. M. Spillane, and K. J. Vahala, Appl. Phys. Lett. 86, 091114 (2005).

5. H. W. Leverenz, Introduction to Luminescence of Solids (Wiley, 1950).

6. F. Auzel, Chem. Rev. 104, 139 (2004).

7. R. G. Smart, D. C. Hanna, A. C. Tropper, S. T. Davey, S. F. Carter, and D. Szebesta, Electron. Lett. 27, 1307 (1991).

8. J. Y. Allain, M. Monerie, and H. Poignant, Electron. Lett. 26, 261 (1990).

9. S. G. Grubb, K. W. Bennett, R. S. Cannon, and W. F. Humer, Electron. Lett. 28, 1243 (1992).

10. W. von Klitzing, E. Jahier, R. Long, F. Lissillour, V. Lefèvre-Seguin, J. Hare, J.-M. Raimond, and S. Haroche, J. Opt. B 2, 204 (2000).

11. T. J. Kippenberg, J. Kalkman, A. Polman, and K. J. Vahala, Phys. Rev. A 74, 051802 (2006).

12. D. K. Armani, T. J. Kippenberg, S. M. Spillane, and K. J. Vahala, Nature 421, 925 (2003).

13. M. J. A. de Dood, L. H. Slooff, A. Polman, A. Moroz, and A. van Blaaderen, Phys. Rev. A 64, 033807 (2001).

14. E. Snoeks, P. G. Kik, and A. Polman, Opt. Mater. 5, 159 (1996).

15. C. B. Layne and M. J. Weber, Phys. Rev. B 53, 6065 (1977).

16. M. L. Gorodetsky and V. S. Ilchenko, J. Opt. Soc. Am. B 16, 147 (1999).

17. M. Cai, O. Painter, and K. J. Vahala, Phys. Rev. Lett. 85, 74 (2000).

18. M. Cai, O. Painter, K. J. Vahala, and P. C. Sercel, Opt. Lett. 25, 1430 (2000).

19. T. Carmon and K. J. Vahala, Nat. Phys. 3, 430 (2007).

20. M. Oxborrow, IEEE Trans. Microwave Theory Tech. 55, 1209 (2007).

21. B. Min, L. Yang, and K. J. Vahala, Phys. Rev. A 76, 013823 (2007).

22. P. E. Barclay, K. Srinivasan, O. Painter, B. Lev, and H. Mabuchi, Appl. Phys. Lett. 89, 131108 (2006). 